\title{
Experimental Bounds on Masses and Fluxes of Nontopological Solitons
}

\author{
J. Arafune and T. Yoshida \\ Institute for Cosmic Ray Research, University of Tokyo \\ Kashiwanoha 5-1-5, Kashiwa 277-8582, Japan \\ S.Nakamura \\ Faculty of Engineering , Yokohama National University \\ Tokiwadai 79-5, Hodogaya-Ku, Yokohama 240-8501, Japan \\ K.Ogure \\ Department of Physics, Kobe University \\ Rokkoudaicho 1-1, Nada-Ku, Kobe 667-8501, Japan
}

(October 26, 2018)

\begin{abstract}
We have re-analyzed the results of various experiments which were not originally interested as searches for the Q-ball or the Fermi-ball. Based on these analyses, in addition to the available data on Q-balls, we obtained rather stringent bounds on flux, mass and typical energy scale of Q-balls as well as Fermi-balls. In case these nontopological solitons are the main component of the dark matter of the Galaxy, we found that only such solitons with very large quantum numbers are allowed. We also estimate how sensitive future experiments will be in the search for Q-balls and Fermi-balls.
\end{abstract}

\section{INTRODUCTION}

In quantum field theory there exist "nontopological solitons" such as Q-balls [1,2], Fermi-balls [3, 1], and neutrinoballs [ [], the stabilities of which are based on conservation of global U(1) charges, rather than on topological quantum numbers. For example, Q-balls are stabilised by the conservation of a U(1) charge [1,2] of scalar fields, while Fermiballs and neutrino-balls are stabilised by the conservation of the number of such fermions that have Yukawa couplings with scalar fields [3 5]. If such nontopological solitons exist, they may solve or at least be closely related to important problems in cosmology such as dark matter [3, 何, the baryon number asymmetry of the universe [2,6 8], and gamma-ray bursts $[5.9$.

Although the ideas of such nontopological solitons are very attractive, the qualitative properties of them, mass scale, charge size, typical energy scale, and cosmic abundance, are so ambiguous that there are many orders of magnitude in the parameter space that must be considered. It is desirable to make the parameter regions of nontopological solitons as narrow as possible through the use of currently available observational data. This will clarify the regions that are to be searched through experimentation in the near future. Although there are reports of a number of useful observational or phenomenological analyses to examine the allowed parameter regions of Q-balls, there does not yet exist an available analysis. In fact, Kusenko et al. 10 discussed the experimental signatures of Q-balls and pointed out powerful detection methods for neutral Q-balls. The Gyrlyanda experiments at Lake Baikal [11] reported the flux limit of neutral Q-balls applying these methods to their monopole search experiments. The report also gave rough estimates of bounds to be obtained with other monopole search experiments, "Baksan" scintillators [12] and old aged mica 13. Bakari et al. 14. calculated the energy losses of Q-balls in matter and concluded the various MACRO detectors can search for charged Q-balls \$. When it comes to Fermi-balls, no experimental limits on the flux have been reported to date.

In this paper, we comprehensively discuss the flux limits of Q-balls and Fermi-balls. As their mass could be very large, we use various kinds of results from direct searches for supermassive magnetic monopoles [15], nuclearites, and/or heavy primary cosmic rays [16]. We also estimate the sensitivity of present and future experiments for the purpose of searching for nontopological solitons. While these experiments were mainly designed for different purposes, we stress their importance in the specific analysis of nontopological solitons. In the following discussion, we analyze the flux bounds taking the charge $Z_{Q}$ for the case of $Z_{Q}=0,1,2,3,10$, and 137 , as typical values. If the charge is larger than 137, the Q-ball will have the electromagnetic properties at low energy similar to the case of $Z_{Q}=137$, having a geometrical cross section $\pi R_{Q}^{2}$ with the effective radius of at least $R_{Q} \sim 1 \AA$ [19]. For the case of Fermiballs, we assume that the electric charge to be large enough in order to assure its stability against deformation and

\footnotetext{
1 The explicit values of the flux upper limits on Q-balls are not available yet.
} 
fragmentation [4], and that the electromagnetic properties at low energy is the same as the case of $Z_{F}=137$ unless the radius exceeds $1 \AA$.

\section{EXPERIMENTAL BOUNDS ON Q-BALL}

\section{A. Q-ball Properties}

In the following our arguments will be restricted to the case of thick wall Q-balls, since most attractive Q-balls in SUSY are of this case [2]. We briefly review the properties of Q-balls in order to clarify our notations and assumptions in estimating the flux bounds.

Q-balls consist of a complex scalar field with a conserved global U(1) charge. The scalar field, $\varphi$ vanishes outside the Q-ball, while it forms a coherent state inside with a time-dependent phase [1],

$$
\varphi(t, \vec{x})=\varphi(\vec{x}) e^{-i \omega t}
$$

In this circumstance, the Q-ball is at the lowest energy state with a fixed global U(1) charge. This is derived by minimizing the Q-ball mass $M_{Q}$,

$$
M_{Q}=\int d x\left[\left|\partial_{t} \varphi\right|^{2}+|\vec{\nabla} \varphi|^{2}+V\left(|\varphi|^{2}\right)\right],
$$

under the constraint on the charge $Q$,

$$
Q=i \int d x\left[\varphi^{*} \partial_{t} \varphi-\varphi \partial_{t} \varphi^{*}\right]
$$

Here we assume 2 that for large $\varphi$ the scalar potential $V\left(|\varphi|^{2}\right)$ is almost "flat", i.e., $V\left(|\varphi|^{2}\right) \simeq M_{S}^{4}$, where $M_{S}$ is a constant with a mass dimension. (The potential of this type is known to be present in supersymmetric theories, in which $M_{S}$ is a SUSY breaking scale). In this case [10], the Q-ball mass is obtained as

$$
M_{Q}=\frac{4 \pi \sqrt{2}}{3} M_{S} Q^{3 / 4},
$$

with its radius,

$$
R_{Q}=\frac{1}{\sqrt{2}} M_{S}^{-1} Q^{1 / 4}
$$

The proportionality, $M_{Q} \propto Q^{3 / 4}$, in Eq.(4) leads to the stability of the Q-ball for $Q$ large enough. If we consider a baryonic Q-ball, i.e., a B-ball, 3 for example, the condition of stability against nucleon emission is $m_{\text {proton }}>\frac{\partial}{\partial Q} M_{Q}$, i.e.,

$$
Q>5.0 \times 10^{14}\left(\frac{M_{S}}{\mathrm{TeV}}\right)^{4} .
$$

It is pointed out that such large Q-balls may have been created in the early universe [8,6, 17]. If this is the case and such large stable Q-balls have survived until present day, they would contribute toward the dark matter in the Galaxy. Their flux should then satisfy

\footnotetext{
${ }^{2}$ We stress that our result does not lose generality by making this assumption. The experimental flux limits, which we obtain later as a function of mass of the Q-ball, are independent of this assumption in case where Q-balls are charged. This is due to the fact that it solely depends on the mass of the Q-ball. However, in the case of neutral Q-balls, they are dependent on this assumption, since they rely only on their cross section rather than mass. Of course, the flux limitations of neutral Q-balls were independent of the assumption if we expressed them as a function of the cross section. Readers, who are interested in neutral Q-balls with another type of potential, can easily accommodate our results by matching the mass of the Q-balls with such of ours, that has the same cross section as them.

${ }^{3} \mathrm{~A}$ B-ball is the Q-ball the conserved $\mathrm{U}(1)$ charge of which is baryon number.
} 


$$
F \leq F_{D M} \sim \frac{\rho_{D M} v}{4 \pi M_{Q}} \sim 7.2 \times 10^{5}\left(\frac{\mathrm{GeV}}{M_{Q}}\right) \mathrm{cm}^{-2} \mathrm{sec}^{-1} \mathrm{sr}^{-1},
$$

where $\rho_{D M}$ is the energy density of the dark matter in the Galaxy, $\rho_{D M} \sim 0.3 \mathrm{GeV} / \mathrm{cm}^{3}$, and $v$ is the Virial velocity of the Q-ball, $v \sim 3 \times 10^{7} \mathrm{~cm} / \mathrm{sec}$. In the following analysis for Q-balls and Fermi-balls, we assume for simplicity the velocity of Q-balls to be $v=10^{-3} c$, where $c$ is the light velocity.

In order to estimate efficiencies in the detection of Q-balls with various detectors, Kusenko et al. 10 classified relic solitons into two groups according to the properties of their interaction with matter: Supersymmetric Electrically Neutral Solitons(SENS) and Supersymmetric Electrically Charged Solitons(SECS). In this paper we use the terms "SENS" and "SECS" only for Q-balls. In case of SENS, a process similar to proton decay may occur in the thin layer of the Q-ball's surface, when the Q-ball collides with nuclei. The energy release of roughly $1 \mathrm{GeV}$ per nucleon is carried away by pions through this process. We call this the "KKST process". In case of SECS, with a positive charge, the KKST process in collision with nuclei will be strongly suppressed by Coulomb repulsion. Only electromagnetic processes will take place in this circumstance. A charged Q-ball with a small velocity is accompanied by an atomiclike cloud of electrons and interacts with matter similarly to an atom with a heavy mass. If the charge is very large, the effective interaction radius is approximately $1 \AA$, which is similar to the case of "nuclearites" [18, 19]. Negatively charged Q-balls are of little interest to us at present. If negatively charged Q-balls happen to exist, the detection of them should be much easier than that of positively charged Q-balls. This is due to both the electromagnetic process and the KKST process occurring during collision. As a result of this, the excluded parameter region of the positively charged Q-ball is also excluded for the negatively charged ones. For the purpose of this paper, we will only discuss the neutral and positively charged Q-balls.

\section{B. Bounds on Flux and Mass of Neutral Q-ball (SENS)}

As pointed out by Kusenko et al. [10], a neutral Q-ball would produce the KKST process, when it collides with a nucleus, absorbing it and emitting pions with the total energy of approximately $1 \mathrm{GeV}$ per nucleon. If the cross section of such a process is large and successive events of this type are detected along a single trajectory, it will be a signal detecting a Q-ball. We note that such a Q-ball process is different from the Rubakov effect [20] even though they are similar to each other. The cross section for the former process depends little on whether the target nucleus possesses a magnetic moment or not, while the cross section for the latter process strongly depends on it. T Since the interaction cross section of the KKST process is expected of a geometrical size, the energy loss of SENS is determined by

$$
\frac{d E}{d x}=-\pi R_{Q}^{2} v^{2} \rho,
$$

similar to the case of nuclearite collision with a nucleus 18,19. Here $R_{Q}$ and $\rho$ are the radius of the Q-ball and the density of the target matter, respectively. In order for the Q-balls to reach the detector site and penetrate the detector, the following condition should be satisfied as:

$$
M_{Q} \gtrsim \frac{1}{7.3} \pi R_{Q}^{2} \int \rho d x
$$

with integration over the trajectory of Q-balls. This leads to

$$
M_{Q} \gtrsim 4.2 \times 10^{-39}\left(\frac{\rho L}{\mathrm{gr} \mathrm{cm}^{-2}}\right)^{3}\left(\frac{\mathrm{TeV}}{M_{S}}\right)^{8} \mathrm{GeV},
$$

where $L$ is the path length for the Q-ball to traverse matter (air, water, and/or rock) and to penetrate the detector. Since we are interested in the parameter regions of $M_{S}>100 \mathrm{GeV}, \rho \leq 10 \mathrm{gr} \mathrm{cm}^{-3}, L \leq 2 R_{\text {Earth }} \sim$ $1.3 \times 10^{10} \mathrm{~cm} \mathrm{gr} \mathrm{cm}^{-2}$ and $M_{Q} \gg M_{S}$, this condition is always satisfied in any experiments on the earth.

Most experimental searches for monopole-catalyzed proton decay (the Rubakov effect [20]) are also sensitive to the KKST process, and are able to give stringent bounds on SENS flux. Let us review the available data, not only

\footnotetext{
${ }^{4}$ If the nucleus has a finite magnetic moment, the cross section of the Rubakov process should have an enhancement factor 21] of $\sim 1 / \beta^{2} \sim 10^{6}$ with $\beta$ being the relative velocity (in unit of the light velocity) between the monopole and the nucleus.
} 
the already reported results of the Gyrlyanda experiments at Lake Baikal on SENS flux [1] but also other typical experiments reinterpreting them to get SENS flux bounds.

The Gyrlyanda experiments reported that the flux of SENS has the bound [11]:

$$
F<3.9 \times 10^{-16} \mathrm{~cm}^{-2} \mathrm{sec}^{-1} \mathrm{sr}^{-1}
$$

if the cross section for the KKST process is $\sigma>1.9 \times 10^{-22} \mathrm{~cm}^{2}$. This corresponds to the lower limit of the SENS mass,

$$
M_{Q}>1.0 \times 10^{21}\left(\frac{M_{S}}{\mathrm{TeV}}\right)^{4} \mathrm{GeV}
$$

since Eq.(14) and Eq.(5) relate $\sigma$ to $M_{Q}$ as

$$
\sigma=1.9 \times 10^{-36}\left(\frac{\mathrm{TeV}}{M_{S}}\right)^{8 / 3}\left(\frac{M_{Q}}{\mathrm{GeV}}\right)^{2 / 3} \mathrm{~cm}^{2} .
$$

The authors of Ref. [11] also estimated the SENS flux limit which will be given in the BAKSAN experiments [12]:

$$
F<3.0 \times 10^{-16} \mathrm{~cm}^{-2} \mathrm{sec}^{-1} \mathrm{sr}^{-1},
$$

if the cross section is $\sigma>5.0 \times 10^{-26} \mathrm{~cm}^{2}$, which corresponds to

$$
M_{Q}>4.2 \times 10^{15}\left(\frac{M_{S}}{\mathrm{TeV}}\right)^{4} \mathrm{GeV} .
$$

Although the above two experiments give considerably stringent bounds to SENS, we must examine how stringent limits will be given by other monopole searches and future cosmic ray experiments.

The Kamiokande Cherenkov detector [22], which has 3000 tons of water, approximately 1000 photo-tubes and is located at approximately 1000 meters underground, gave the limits of monopole with 335 days of live time. We reinterpret the results to obtain SENS flux limits approximately [34]:

$$
F<3 \times 10^{-12}, \quad 3 \times 10^{-14} \text {, and } 3 \times 10^{-15} \mathrm{~cm}^{-2} \mathrm{sec}^{-1} \mathrm{sr}^{-1},
$$

for SENS with $\sigma=0.1,1$, and $10 \mathrm{mb}$, which correspond to

$$
M_{Q}=4.0 \times 10^{11}, 1.2 \times 10^{13}, \text { and } 5.6 \times 10^{13}\left(\frac{M_{S}}{\mathrm{TeV}}\right)^{4} \mathrm{GeV},
$$

respectively.

The Super-Kamiokande experiments with 50000 tons of water, the Cherenkov detector would obtain more stringent flux limits than those of Kamiokande by almost two orders of magnitude and with three years of observation time.

The MACRO, large underground detectors 14,23,24 consisting of three kinds of subdetectors ( i.e. scintillation counters, streamer tubes, and nuclear track detectors(CR-39)) could search for SENS 114]. Although the flux limits of SENS are reported to be obtained as $F \lesssim 10^{-16} \mathrm{~cm}^{-2} \mathrm{sec}^{-1} \mathrm{sr}^{-1}$ in Ref. [14], we do not give this flux limit here since the detection efficiency is too difficult for us to estimate.

The AMANDA Cherenkov detectors [25,26] located at the South Pole under ice, are designed mainly to detect relativistic particles. If they could also detect slow particles, the large area of the array would be of great help. For a few years to observe SENS, they could obtain their flux limits as $F \lesssim 10^{-17} \mathrm{~cm}^{-2} \mathrm{sec}^{-1} \mathrm{sr}^{-1} / \epsilon$ with the detection efficiency, $\epsilon$. The flux limits, however, are not estimated here since the detection efficiencies are also difficult for us to evaluate.

The TA(Telescope Array Project) with effective aperture of $S \Omega \epsilon=6 \times 10^{3} \mathrm{~km}^{2} \mathrm{sr}$ $\left(S \Omega=6 \times 10^{4} \mathrm{~km}^{2} \mathrm{sr}\right.$ and the duty factor $\left.\epsilon=0.1\right)$ is planned to detect cosmic rays with energy, $10^{16}-10^{20} \mathrm{eV}$, by detecting the air fluorescence [27]. Using a special trigger, it may detect slow particles with large energy loss [28]. With such a trigger, it may be possible to search for SENS flux at the level of $F<1 \times 10^{-21} \mathrm{~cm}^{-2} \mathrm{sec}^{-1} \mathrm{sr}^{-1}$, provided that the fluorescence light yield of the event is equivalent to that of air showers with the minimum energy $E_{\min }=10^{16} \mathrm{eV}$. Considering energy release of roughly $1 \mathrm{GeV}$ per absorbed nucleon in the KKST process, one obtains the condition of detectability in $T A$ : 


$$
\sigma \int \rho d x \geq E_{\min } / \xi_{S E N S}
$$

where $\xi_{S E N S}$ is the ratio of the efficiency of fluorescence light yield per total energy loss of SENS to that of extensive air showers. In the KKST process we estimate $\xi_{S E N S} \sim 1$, since emitted pions are relativistic. Assuming the average density of air $\rho \sim 0.5 \times 10^{-3} \mathrm{~g} / \mathrm{cm}^{3}$ and the path length of SENS in the atmosphere $L \sim 20 \mathrm{~km}$, we obtain the lower limit of SENS mass from Eq.(18),

$$
M_{Q}>0.7 \times 10^{24}\left(\frac{M_{S}}{\mathrm{TeV}}\right)^{4} \mathrm{GeV} .
$$

The $O A\left(O W L\right.$-AIRWATCH) detector [42] located on a satellite with effective aperture $5 \times 10^{5} \mathrm{~km}^{2}$ sr (with duty cycle of 0.2 taken into account), is used to observe the atmospheric fluorescence of air showers with energy $10^{19}-10^{20}$ $\mathrm{eV}$ or more. If it could also detect slow particles with high efficiency, it would be possible to search for SENS during a one year time constraint at the flux level of $F<10^{-23} \mathrm{~cm}^{-2} \mathrm{sec}^{-1} \mathrm{sr}^{-1}$. For SENS to yield the amount of fluorescence light comparable to that of air showers with $10^{19} \mathrm{eV}$, their mass should satisfy $M_{Q}>2 \times 10^{28}\left(\frac{M_{S}}{\mathrm{TeV}}\right)^{4} \mathrm{GeV}$.

The bounds given in the various experiments mentioned above are summarized in Fig.1 (a). This figure also gives the flux to be expected if the dark matter of the Galaxy consists mainly of SENS(see Eq.(可)). Note that these bounds of excluded regions depend on $M_{S}$. ( The lower mass bound in each experiment is calculated taking $M_{S}=1 \mathrm{TeV}$ in this figure. In case of $T A$ and $O A$, we added those for $M_{S}=100 \mathrm{GeV}$. ) The region in which it is expected that future experiments are able to be conducted, are also given there. If we assume that the dark matter of the Galaxy consists mainly of SENS ( i.e. if the flux is just on the DM-limit line of Fig.11(a)), we obtain the excluded regions of SENS mass as functions of the parameter $M_{S}$ as in Fig. 1. (b). This figure shows that SENS with the U(1) charge $Q \gtrsim 10^{22}$ are cosmologically interesting in the region of $M_{S} \gtrsim 6 \times 10^{3} \mathrm{GeV}$, while that $T A$ and $O A$ could search for SENS of $10^{25} \lesssim Q \lesssim 10^{35}$ in the region of $M_{S} \lesssim 6 \times 10^{3} \mathrm{GeV}$.

\section{Bounds on Flux and Mass of Charged Q-ball (SECS)}

Charged Q-balls (SECS) interact with matter in a similar way to nuclearites [18,19]. The rate of energy loss of SECS in matter is

$$
\frac{d E}{d x}=-\sigma \rho v^{2},
$$

where $\sigma$ is the cross section of SECS collision with matter, $\rho$ is the density of the matter, and $v$ is the relative velocity of SECS and the matter. Substituting $E=\frac{1}{2} M_{Q} v^{2}$ in the above equation and integrating with respect to $x$, one obtains

$$
\rho L=\frac{M_{Q}}{\sigma} \ln \frac{v_{0}}{v_{c}}
$$

where $L$ is the range of SECS in the medium, $v_{0}$ is an initial velocity of $\operatorname{SECS}\left(\sim 3 \times 10^{7} \mathrm{~cm} / \mathrm{sec}\right)$, and $v_{c}$ is a final velocity of SECS. We use the estimation in the case of nuclearites, $v_{c} \sim 1.7 \times 10^{4} \mathrm{~cm} / \mathrm{sec}$ in rock 19]. SECS with a velocity below this value are quickly brought to rest. In the case of SECS with a radius larger than $1 \AA$ (i.e., $\left.M_{Q}>2.1 \times 10^{30}\left(M_{S} / \mathrm{TeV}\right)^{4} \mathrm{GeV}\right)$, the cross section is simply given by $\sigma=\pi R_{Q}^{2}$. From Eqs.(蛋), (5) and (21), we obtain

$$
\rho L=6.2 \times 10^{12}\left(\frac{M_{S}}{\mathrm{TeV}}\right)^{8 / 3}\left(\frac{M_{Q}}{\mathrm{GeV}}\right)^{1 / 3} \mathrm{gr} / \mathrm{cm}^{2} .
$$

Such heavy SECS should, however, have too small a flux to be detected according to Eq.(7), and thus, are not interesting to us. We have considered only SECS with a radius smaller than $1 \AA$ in the following.

If such SECS have a significantly large electric charge $\left(Z_{Q} \gtrsim \alpha^{-1} \sim 137\right.$ where $\alpha$ is the fine structure constant), the cross section of their collision with matter is not controlled by their intrinsic radius $R_{Q}$, but by the size of the surrounding electron cloud. This cloud is never smaller than $\sim 1 \AA$. We note that this situation is similar to the case of nuclearites [19]. We have $\sigma=\pi R_{\text {eff }}^{2}$ with $R_{\text {eff }}=1 \AA$, which leads to the relation

$$
\rho L=4.0 \times 10^{-8}\left(\frac{M_{Q}}{\mathrm{GeV}}\right) \mathrm{gr} / \mathrm{cm}^{2} .
$$


Equation (23) shows that SECS with $Z_{Q} \gtrsim 137$ should be as heavy as

$$
M_{Q}>2.5 \times 10^{7}\left(\frac{\rho L}{\mathrm{gr} / \mathrm{cm}^{2}}\right) \mathrm{GeV}
$$

in order to penetrate the medium with density $\rho$ and length $L$.

If the charge of SECS is small, i.e. $Z_{Q} \ll 137$ (and the intrinsic SECS radius $R_{Q}$ is also smaller than $1 \AA$ ), the effective cross section should be smaller than $\pi(1 \AA)^{2}$. We then need more delicate treatments to estimate the rate of the energy loss. It is known that there are two kinds of interaction that contribute to the energy loss of SECS at low velocity $\beta \sim 10^{-3}$ ( i.e. interaction with electrons and interaction with nuclei ), $d E / d x=(d E / d x)_{\text {electrons }}+(d E / d x)_{\text {nuclei }}$ 14,29 33. The rate of electronic energy loss is estimated as Ref. [29],

$$
\left(\frac{d E}{d x}\right)_{\text {electrons }}=8 \pi \alpha a_{0} \frac{v}{v_{0}} N_{e} \frac{Z_{Q}^{7 / 6}}{\left(Z_{Q}^{2 / 3}+Z^{2 / 3}\right)^{3 / 2}},
$$

where $v_{0}$ is given by $\alpha c, N_{e}$ is the number density of electrons in the medium, $Z$ is the atomic number of the medium, and $a_{0}$ is the Bohr radius. The rate of energy loss caused by interaction with the nuclei of the medium is given in Ref. [32]

$$
\left(\frac{d E}{d x}\right)_{\text {nuclei }}=4 \pi \alpha a N_{Z} Z_{Q} Z \frac{M_{Q}}{M_{Q}+M} \frac{A \ln B \epsilon}{B \epsilon-(B \epsilon)^{-C}},
$$

with $A=0.56258, B=1.1776, C=0.62680, M$ is the mass of the target nucleus, $N_{Z}$ is the number density of target nuclei and

$$
\epsilon=\frac{a M M_{Q} \beta^{2}}{2 \alpha Z_{Q} Z\left(M_{Q}+M\right)}, \quad a=\frac{0.8853 a_{0}}{\left(\sqrt{Z_{Q}}+\sqrt{Z}\right)^{2 / 3}} .
$$

In case of $M_{Q} \gg M$ we calculated $(d E / d x)_{\rho} \equiv(d E / d x) / \rho$ (which depends only loosely on the density of the medium) for $\mathrm{SiO}_{2}$ and obtained $(d E / d x)_{\rho}=0.16,0.41,0.71$, and $2.9 \mathrm{GeV} / \mathrm{gr} / \mathrm{cm}^{2}$ for SECS with typical charge $Z_{Q}=1,2,3$, and 10, respectively. We also find the energy loss rate $(d E / d x)_{\rho}$ depends little on matter medium(air, water, and/or rock). We use the above values for the following estimation of energy loss. The condition for SECS to penetrate the medium with density $\rho$ and length $L$ is roughly given by

$$
\frac{1}{2} M_{Q} \beta^{2}>\left(\frac{d E}{d x}\right)_{\rho} \rho L .
$$

Since SECS have a large cross section when they collide with matter, they should have kinetic energy large enough to reach a detector and to penetrate it. This situation is unlike that of SENS. Let us take the following experiments as typical in detecting SECS.

The MACRO with its three subdetectors are sensitive to SECS [14,23,24] for any values of $Z_{Q}$; in order for SECS to reach the underground detector site from above with $\rho L \sim 3.7 \times 10^{5} \mathrm{gr} \mathrm{cm}^{-2}$, the lower limit of the SECS mass $M_{Q}$ is given by Eq.(24) and Eq.(28), as $M_{Q}>1.2 \times 10^{11}, 3.0 \times 10^{11}, 5.3 \times 10^{11}, 2.1 \times 10^{12}$, and $9.3 \times 10^{12} \mathrm{GeV}$ for $Z_{Q}=1,2,3,10$, and 137, respectively. The flux limit suggested by the results of no events of monopole search experiments [14] is roughly [34] $F \lesssim 10^{-16} \mathrm{~cm}^{-2} \mathrm{sec}^{-1} \mathrm{sr}^{-1}$. It is obvious that the inclusion of searches for Q-balls from below, decreases the flux upper limit by a factor of two, although the lower limits for the mass to reach the detector increase by a factor of $(\rho L)_{\text {from below }} /(\rho L)_{\text {from above }}=\left(6.6 \times 10^{9}\right) /\left(3.7 \times 10^{5}\right) \sim 1.8 \times 10^{4}$. This feature for SECS is common to other experiments such as OYA, NORIKURA, KITAMI, AKENO, UCSDII, KEK, and MICA.

The $O Y A$ experiments [35] with CR-39 plastic track detectors (located at the depth of $\rho L=10^{4} \mathrm{~cm}^{-2} \mathrm{sec}^{-1} \mathrm{sr}^{-1}$ ), searched for monopoles and nuclearites for 2.1 years. The flux limit of these experiments corresponds to that of SECS, $F<3.2 \times 10^{-16} \mathrm{~cm}^{-2} \mathrm{sec}^{-1} \mathrm{sr}^{-1}$ [34]. Since the detectors are sensitive to the restricted energy loss larger than $\sim 0.14 \mathrm{GeV} \mathrm{gr} / \mathrm{cm}^{2}$, it is possible that SECS with a charge of $Z_{Q} \geq 2$ may be detected. The condition for SECS to reach the detector is $M_{Q}>8.2 \times 10^{9} \mathrm{GeV}, 1.4 \times 10^{10} \mathrm{GeV}, 5.8 \times 10^{10} \mathrm{GeV}$, and $2.5 \times 10^{11} \mathrm{GeV}$ for $Z_{Q}=2,3,10$, and 137 , respectively.

The KEK experiments with scintillation counters placed at ground level [37] should be sensitive to SECS at any value of $Z_{Q}$. As the detection threshold is $0.01 I_{\min }$ where $I_{\min }$ is the minimum energy loss of the ionizing particle with $Z=1$ [34]. We interpret that their flux upper limit for strange quark matter (nuclearites) holds for the SECS: 
$F<3.2 \times 10^{-11} \mathrm{~cm}^{-2} \mathrm{sec}^{-1} \mathrm{sr}^{-1}$. The mass bounds for SECS to reach detectors located at ground level are given by $M_{Q}>3.1 \times 10^{8} \mathrm{GeV}, 8.2 \times 10^{8} \mathrm{GeV}, 1.4 \times 10^{9} \mathrm{GeV}, 5.8 \times 10^{9} \mathrm{GeV}$, and $2.5 \times 10^{10} \mathrm{GeV}$ for $Z_{Q}=1,2,3,10$, and 137 , respectively.

The KITAMI experiments with CN (cellulose nitrate) nuclear track detectors installed in houses at sea level, give the flux limit $F<5.2 \times 10^{-15} \mathrm{~cm}^{-2} \mathrm{sec}^{-1} \mathrm{sr}^{-1}[36]$. We reinterpret this as the SECS flux limit [34]. Since the ionization energy loss of SECS should be larger than $\sim 1.3 \mathrm{GeV} / \mathrm{gr} / \mathrm{cm}^{2}$ for a chemically etchable track to be made in CN, we estimate that SECS with $Z_{Q} \geq 10$ can be detected with the CN detectors. At ground level with $\rho L=10^{3}$ gr $\mathrm{cm}^{-2}$, SECS heavier than $5.8 \times 10^{9} \mathrm{GeV}$ and $2.5 \times 10^{10} \mathrm{GeV}$ can penetrate the atmosphere and reach the detector from above. This is the case for $Z_{Q}=10$ and 137 , respectively.

The AKENO experiments with Helium-gas counters 38 located at ground level should be sensitive to SECS at any value of $Z_{Q}$. The detector is composed of many layers of proportional counters and concrete shields. The detector is sensitive to tracks with an ionization level larger than $10 I_{\mathrm{mim}}$. This should therefore assure the detectability for SECS with any value of $Z_{Q}$. SECS with masses of $M_{Q}>5.8 \times 10^{8} \mathrm{GeV}, 1.5 \times 10^{9} \mathrm{GeV}, 2.6 \times 10^{9} \mathrm{GeV}, 1.1 \times$ $10^{10} \mathrm{GeV}$, and $4.7 \times 10^{10} \mathrm{GeV}$, can penetrate the earth and the concrete shields of the detector from above for $Z_{Q}=1,2,3,10$, and 137, respectively. Due to the fact that no events have been detected, we estimate the flux limit as $F<1.8 \times 10^{-14} \mathrm{~cm}^{-2} \mathrm{sec}^{-1} \mathrm{sr}^{-1} 34$.

The UCSDII experiments with $\mathrm{He}-\mathrm{CH}_{4}$ proportional tubes placed at ground level [39], should be sensitive to SECS with any value of $Z_{Q}$, as the detection threshold is $0.09 I_{m i m}$. The flux upper limit for monopoles $F=$ $1.8 \times 10^{-14} \mathrm{~cm}^{-2} \mathrm{sec}^{-1} \mathrm{sr}^{-1}$ should be the same as that for SECS [34. The mass bounds are the same as in the above case of the $K E K$ experiments.

The NORIKURA CR-39 experiments were conducted at the top of Mt.Norikura in the search for monopoles and strange quark matter 40]. They are sensitive to tracks with ionization larger than $0.35 \mathrm{GeV} / \mathrm{gr} / \mathrm{cm}^{2}$. This assures that SECS with $Z_{Q} \geq 3$ will be detectable. Since the detector is installed at a high altitude, it is more sensitive to lighter SECS compared to detectors such as those used by KITAMI, AKENO, UCSDII, and KEK, which are installed at ground level. The flux limit is $F=2.2 \times 10^{-14} \mathrm{~cm}^{-2} \mathrm{sec}^{-1} \mathrm{sr}^{-1}$.

The MICA analysis with ancient mica crystals of $0.6-0.9 \times 10^{9}$ years old, was made to search for monopoles 13 . It should also be sensitive to SECS with $Z_{Q} \gtrsim 10$ as the detection threshold is $2.4 \mathrm{GeV} / \mathrm{gr} / \mathrm{cm}^{2}$ [34. In order for SECS coming from above to reach the mica crystals located at $3 \mathrm{~km}$ underground with $\rho L=7.5 \times 10^{5} \mathrm{gr} / \mathrm{cm}^{2}$, SECS should be heavier than $4.4 \times 10^{12} \mathrm{GeV}$ and $1.9 \times 10^{13} \mathrm{GeV}$ for $Z_{Q}=10$ and 137 , respectively. In case of the monopole search, the capture of an aluminium atom by a monopole was taken into account. The detection efficiency was estimated at $\sim 0.15$ for monopoles coming from above and 0 for those from below, due to this consideration. Therefore, this decrease of efficiency caused the flux limits to be less stringent. In case of SECS search, however, SECS need not capture an aluminum atom and can be heavy enough to penetrate the earth. With these considerations we estimate the flux limit for SECS, which is better than that for monopoles by a factor of 6 , to have $F=2.3 \times 10^{-20} \mathrm{~cm}^{-2} \mathrm{sec}^{-1} \mathrm{sr}^{-1}$ for SECS from above and a value improved by a factor of twelve for SECS from any direction.

The $S K Y L A B$ experiments with Lexan track detectors (installed in the SKYLAB workshop located in space), reported that no superheavy relativistic nuclei $(Z \gtrsim 110)$ 16 had been detected, which correspond to $Z_{Q} \gtrsim 10$ in our case of slow particles. The Lexan detector and its container have a thickness $\rho L=2 \mathrm{gr} \mathrm{cm}^{-2}$ in total, or effectively $\rho L \sim 3 \mathrm{gr} \mathrm{cm}^{-2}$, giving the mass a lower limit of SECS $M_{Q}>1.7 \times 10^{7} \mathrm{GeV}$ and $7.5 \times 10^{7} \mathrm{GeV}$ for $Z_{Q}=10$ and 137 , respectively. This experiment gives the flux limit of SECS as $F<3.8 \times 10^{-12} \mathrm{~cm}^{-2} \mathrm{sec}^{-1} \mathrm{sr}^{-1}$ [34].

The $A M S$ experiments may detect SECS in the future if a special trigger designed to detect slow particles is used [41]. The magnetic spectrometer on the space station would have a large area, allowing us to record the flux upper limit as $F \times 10^{-11} \mathrm{~cm}^{-2} \mathrm{sec}^{-1} \mathrm{sr}^{-1}$ given a one year observation time frame 34. The detection with thickness of $\rho L \sim 10 \mathrm{gr} \mathrm{cm}{ }^{-2}$ needs mass $M_{Q}>3.1 \times 10^{6} \mathrm{GeV}, 8.2 \times 10^{6} \mathrm{GeV}, 1.4 \times 10^{7} \mathrm{GeV}, 5.8 \times 10^{7} \mathrm{GeV}$, and $2.5 \times 10^{8} \mathrm{GeV}$ for $Z_{Q}=1,2,3,10$ and 137 , respectively.

The experimental data and also the future possibilities mentioned above are summarized in Figures2 -6. Figures 2(a)-6(a) represent limits of the flux and the mass for SECS. Figures2(a)-14(a) show that future $A M S$ experiments and current $M A C R O$ experiments are very sensitive to a wide parameter region of mass, charge and flux of SECS. On the other hand, Figure5(a) and Figure6(a) show that the $S K Y L A B$ and $M I C A$ experiments give us a stringent exclusion of the lower and upper region of mass respectively. Figures2(b)-6(b) represent bounds on the U(1) charge $Q$ of SECS, versus the symmetry breaking parameter $M_{S}$ in the case that SECS are mainly contributing to dark matter in the Galaxy. If we assume that SECS are B-balls and impose their condition of stability, these figures show that only SECS with $Q \gtrsim 10^{22-26}$ remain to be considered.

\section{EXPERIMENTAL BOUNDS ON FERMI-BALL}




\section{A. Fermi-ball Properties}

We have investigated which regions are allowed for the flux of Fermi-balls and discuss our findings in this section. The Fermi-ball, which is another kind of nontopological soliton, was first proposed by Macpherson and Campbell [3] as a possibility for explaining the make-up of the dark matter in Our Galaxy. Fermi-balls with a large radius are found to be unstable against deformation and are therfore expected to fragment into very small Fermi-balls [3]. The Fermi-ball then interacts with matter in a weak manner and seems too difficult to be detected. It was then proposed by Morris [4] that Fermi-balls be electrically charged in order to improve its stability against deformation and fragmentation. Although a Fermi-ball with large fermion number is energetically unstable and could fragment into many smaller Fermi-balls, the Coulomb force is expected to suppress the process of fragmentation, increasing the life time of the large Fermi-ball so that it would become more stable. Owing to the electromagnetic interaction, various detectors become sensitive to Fermi-balls. In this paper we have focused on the experimental bounds for charged Fermi-balls rather than on neutral Fermi-balls.

Let us first briefly review the properties of Fermi-balls in order to make our assumptions and terminologies clear. The charged Fermi-ball consists of three components: a scalar field, a large number of electrically charged heavy fermions, and also a large number of electrons (or positrons) which partly compensate for the electric charge of the heavy fermions. (When we consider the stability of Fermi-balls in the following, we assume that the electric charge of the heavy fermions is positive without loss of generality.) Inside the Fermi-ball, the scaler field has the value of

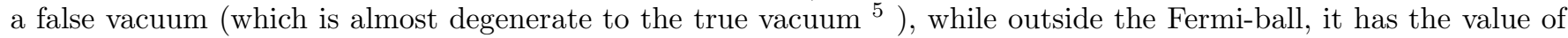
a true vacuum. On the boundary wall, the energy density of the scalar field is higher than those in the two vacua. Since the heavy fermions have a large mass in both the true and false vacuum and have a vanishing mass on the wall, many fermions are strongly trapped on the wall.

Since the electric field of the Fermi-ball is strong, electron-positron pairs may be created by the effect of the quantum field theory. This effect decreases the electric field strength by leaving the electrons on the surface and emitting the positrons to infinity. Assuming the difference of the energy density between two vacua is small enough to be neglected, we obtain the energy of the Fermi-ball with the number of heavy fermions $N_{F}$ and the number of electrons $N_{e}$,

$$
E_{F}=4 \pi \Sigma R^{2}+\frac{2\left(N_{F}^{\frac{3}{2}}+N_{e}^{\frac{3}{2}}\right)}{3 R}+\frac{\alpha\left(N_{F}-N_{e}\right)^{2}}{2 R} .
$$

Here, $\Sigma$ is the surface tension of the Fermi-ball and $R$ is the radius of it. The radius of the stable Fermi-ball, $R_{F}$ is determined by balance ${ }^{5}$ of the surface tension energy (the first term) proportional to $R^{2}$, and the Fermi energy (the second term) or the Coulomb energy (the third term) which is proportional to $R^{-1}$ as

$$
R_{F}=\left\{\frac{1}{8 \pi \Sigma}\left[\frac{2\left(N_{F}^{\frac{3}{2}}+N_{e}^{\frac{3}{2}}\right)}{3}+\frac{\alpha\left(N_{F}-N_{e}\right)^{2}}{2 R}\right]\right\}^{\frac{1}{3}} .
$$

The energy of the Fermi-ball is then,

$$
E_{F}\left(=M_{F}\right)=12 \pi \Sigma R_{F}^{2} \equiv \kappa^{3} R_{F}^{2},
$$

which is the common relationship when the volume energy is neglected. Our following analyses of the Fermi-ball's flux are based only on the above relation. Therefore, the experimental limits from these analyses do not depend on the details of Fermi-ball models.

\section{B. Bounds on Flux and Mass of Fermi-ball}

Throughout this section, we discuss the physical parameter regions that have been excluded in experiments. If the electric charge of the heavy fermions is positive, the observational situation is almost the same as for the case where

\footnotetext{
${ }^{5}$ The approximate degeneracy of energy densities of a true and false vacuum is necessary for the stability of large Fermi-balls.

${ }^{6}$ We have two ways in differentiating Eq.(29) with respect to $R$. : either one fixes $N_{F}$ and $N_{e}$, or one fixes $N_{F}$ and the electric field $\mathcal{E}$ on the surface of the Fermi-ball has the critical value $\mathcal{E}=m_{e}^{2} / e$. Here we take the former and follow Morris 4 . For the other possibility, see Ref. [43].
} 
SECS has $Z_{Q} \gtrsim 137$. This means that the effective radius can be taken as $R_{\text {eff }} \sim 1 \AA$ when the intrinsic radius is smaller than this value. In the case of the Fermi ball (not as is the case with the Q-ball), however, the radius can be larger than $1 \AA$ without becoming too heavy, as its mass is proportional to $R_{F}^{2}$ rather than $R_{F}^{3}$. The effective radius thus becomes,

$$
R_{e f f}= \begin{cases}1 \AA & \left(\text { for } R_{F}<1 \AA\right) \\ R_{F} & \left(\text { for } R_{F} \geq 1 \AA\right) .\end{cases}
$$

In the case where $R_{F}$ is large enough, Fermi-balls can be detected with not only detectors which are sensitive to SECS with $Z_{Q} \gtrsim 137$, but also with future detectors used for extensive air showers such as $T A$ and $O A$. This detectability within future experiments is the main difference in the case of SECS with $Z_{Q} \gtrsim 137$. We examine the bounds on Fermi-balls to be given by AMS, SKYLAB, UCSDII, AKENO, KEK, NORIKURA, KITAMI, OYA, MACRO, $M I C A, T A$, and $O A$ in the following.

First, we discuss the conditions in which the Fermi-ball reaches and penetrates the detectors. For Fermi-balls with $R_{e f f}=1 \AA\left(R_{F}<1 \AA\right)$, the condition is the same as that for SECS with $Z_{Q} \gtrsim 137$, i.e. Eq.(24),

$$
M_{F}>2.5 \times 10^{7}\left(\frac{\rho L}{\mathrm{gr} / \mathrm{cm}^{2}}\right) \mathrm{GeV} .
$$

For Fermi-balls with $R_{e f f}=R_{F}\left(R_{F}>1 \AA\right.$ ), the condition is different from that of SECS (Eq.(22)), since the relation between the mass and the radius of Fermi-balls is different from that of Q-balls. Using Eq.(21) and Eq.(31) with $\sigma=\pi R_{F}^{2}$, we obtain the condition

$$
\kappa \geq 4.7 \times 10^{-2}\left(\frac{\rho L}{\mathrm{gr} / \mathrm{cm}^{2}}\right)^{\frac{1}{3}} \mathrm{GeV} .
$$

This condition is independent of the mass of Fermi-balls in the case of $R_{F} \geq 1 \AA$. From Eq.(33) and Eq.(34) we see that all the experiments (except $T A$ and $O A$ ) give the same bounds on mass and flux of Fermi-balls as on those of SECS with $Z_{Q} \geq 137$, as the detection efficiencies for these two kinds of solitons are the same for these detectors.

We next discuss the conditions for detecting Fermi-balls with $T A$ and $O A$. This requires a fluorescence light yield which corresponds to the air shower energy of $E_{\min }=10^{16} \mathrm{eV}$ for $T A$ [27,28] and $E_{\min }=10^{19} \mathrm{eV}$ for $O A$ [42]. This condition is satisfied if the energy loss of the Fermi-ball is

$$
\pi R_{F}^{2} \rho v^{2} L \geq E_{\min } / \xi_{F} .
$$

Here, the parameter $\xi_{F}$ is the ratio of the efficiency of fluorescence light yield per total energy loss for slow Fermi-balls to that for high energy cosmic ray protons. We estimated $\xi_{F}$ from the measurements of the efficiency of ionization for slow ions [31, 30] as $\xi_{F} \sim 1 / 5$. By taking the average density of air as $\rho=5.0 \times 10^{-4} \mathrm{~g} / \mathrm{cm}^{3}$, the velocity of the Fermi-ball as $v=10^{-3} c$ and the length of the trajectory as $L=20 \mathrm{~km}$, one obtains the required condition to observe Fermi-balls from Eq.(31) and Eq.(35) 8 ,

$$
M_{F} \geq 6.5 \times 10^{22}\left(\frac{\kappa}{10^{3} \mathrm{GeV}}\right)^{3}\left(\frac{E_{\min }}{10^{16} \mathrm{eV}}\right) \mathrm{GeV} .
$$

If $T A$ can observe slow particles 9 , it will be able to search for Fermi-balls on order to give the same flux limit as that of SENS, $F<1 \times 10^{-21} \mathrm{~cm}^{-2} \mathrm{sec}^{-1} \mathrm{sr}^{-1}$ [27]. Equation(36) gives the mass bounds required to observe Fermiballs, $M_{F} \geq 6.5 \times 10^{22}\left(\frac{\kappa}{10^{3} \mathrm{GeV}}\right)^{3} \mathrm{GeV}$. If $O A$ can observe slow particles as well, the flux bounds to be obtained are improved by two orders of magnitude $F<1 \times 10^{-23} \mathrm{~cm}^{-2} \mathrm{sec}^{-1} \mathrm{sr}^{-1}$, 42 , for $M_{F} \geq 6.5 \times 10^{25}\left(\frac{\kappa}{10^{3} \mathrm{GeV}}\right)^{3} \mathrm{GeV}$.

\footnotetext{
${ }^{7}$ In the following, we assume that the electric charge of Fermi-balls is positive. In case Fermi-balls have a negative electric charge, it is much easier for us to detect them than those with a positive charge. This is followed by the fact that the negatively charged Fermi-balls may trap nuclei in collision with matter and emit mesons or photons with total energy of order $1 \mathrm{GeV}$ per nucleon.

We note that all regions excluded for the positively charged Fermi-balls should also be excluded for the negatively charged Fermi-balls.

${ }^{8}$ Here we did not assume the black body radiation from the Fermi-ball trajectory, since it is effective only for dense medium $[19$.

${ }^{9}$ The $T A$ experiments may be available for slow particle search with a special trigger 28
} 
The flux limit with $\kappa=10^{3} \mathrm{GeV}$ is shown in Figure $7(\mathrm{a})$. This figure shows that quite broad regions are already excluded by available experimental data. Future experiments, $T A$ and $O A$, may find it possible to search large regions which have not been previously accessible through current experiments. Figure](b) shows the region of $M_{F}-\kappa$ plane (hatched with solid lines and with half-tone dot meshing) to be excluded when we assume that the dark matter of the Galaxy consists mainly of Fermi-balls.

Here we discuss how these results constrain the Morris's simple Fermi-ball model 伯. The electric field becomes the critical value $\left(\mathcal{E}=m_{e}^{2} / e\right)$, near the surface due to the surrounding electrons in this model. The third term of Eq.(29) is $m_{e}^{4} R^{3} / 2 \alpha$ in this case. When this Coulomb energy is related to the surface energy as $4 \pi \Sigma R^{2}=C m_{e}^{4} R^{3} / 2 \alpha$ we obtain,

$$
M_{F} \sim 3(4 \pi \Sigma)^{3}\left(\frac{2 \alpha}{C m_{e}^{4}}\right)^{2}=6.0 \times 10^{21} C^{-2}\left(\frac{\kappa}{\mathrm{GeV}}\right)^{9} \mathrm{GeV} .
$$

In order to prevent the Fermi-ball from forming a black hole, its radius should be larger than the Schwarzschild radius, $M_{F} / M_{p l}^{2}$, where $M_{p l}$ is the Planck mass. From this condition, we obtain the following constraint of $\kappa$ using Eq.(37),

$$
\kappa \leq 3.3 \times 10^{4} C^{\frac{1}{6}} \mathrm{GeV} .
$$

We draw lines which represent Eq.(37) and Eq.(38) in Figuref(b). We find that the TA and $O A$ experiments are powerful to search the new region of approximately $10^{17} \mathrm{GeV} \lesssim M_{F} \lesssim 10^{29} \mathrm{GeV}$ in Morris's Model (taking $C=1$ ).

\section{CONCLUSION}

Quantum field theory allows the existence of such nontopological solitons as Q-balls and Fermi-balls, the stability of which is supported by the conservation of a global U(1) charge. These solitons may play important roles in cosmology in solving the problems of dark matter, baryogenesis, and gamma ray bursts. In this paper, we have considered Q-balls and Fermi-balls, both of which are typical nontopological solitons.

We have examined the parameter regions, masses, fluxes, charges, and energy scales of Q-balls and Fermi-balls that are to be excluded. These exclusions were derived by analyzing the various existing and future searches for monopoles, nuclearites, and cosmic rays. We also analysed the existing results and analyses of the Q-ball searches of Gyrlyanda and MACRO. The experiments considered here include: (1) underground searches with Gyrlyanda, BAKSAN, Kamiokande, Super-Kamiokande, MACRO, OYA, MICA, and AMANDA; (2) searches on the earth's surface with NORIKURA, KITAMI, KEK, AKENO, UCSDII, and TA; (3) space experiments with SKYLAB, AMS, and OA.

We summarized these experimental data and obtained bounds on the mass and the flux of Q-balls and Fermiballs. Of course, the precise estimation of bounds should be more carefully made by those who did, or will do, the experiments. We believe, however, that our estimations will give useful information in the research of Q-balls and Fermi-balls.

We first investigated Q-balls with an electric charge $Z_{Q}=0$ (SENS) which can be detected through proton-decay like process as proposed in Ref. [10]. We found that a considerably large region $Q \gtrsim 10^{25}$ has already been excluded in $Q-M_{S}$ plane for $M_{S}<100 \mathrm{GeV}$ only by existing experiments (see Figurel (b)), and that a wider region $Q \gtrsim 10^{35}$ could be searched in future experiments of $T A$ and $O A$. We also found that the region $Q=B \gtrsim 10^{22}$ has been excluded for B-balls for any value of $M_{S}$.

We next investigated Q-balls with $Z_{Q}=1,2,3,10$, and 137 (SECS) which interact with matter, mainly by electromagnetic force, just like nuclearites (though the relations between the radius and the mass are quite different). We saw that larger $Z_{Q}$ gives more stringent bounds on flux and mass of SECS. We found that for the value of $M_{S} \sim 10^{2} \mathrm{GeV}$, experimental data gives more stringent limitations on SECS global U(1) charge than the stability condition of B-balls. Q-balls with $Q \gtrsim 10^{22-26}$ still remain to be examined (see Figure226).

We finally investigated Fermi-balls with electric charge $Z_{F} \gg 137$, which are expected to be rather stable against perturbative deformation and fragmentation. We obtained bounds on mass of Fermi-balls, $M_{F} \lesssim 10^{8} \mathrm{GeV}$ and $M_{F} \gtrsim 10^{29} \mathrm{GeV}$ for $\kappa \gtrsim 0.1 \mathrm{GeV}$ in $M_{F}-\kappa$ plane. If we further assume the Morris Model [4], we obtained more

\footnotetext{
${ }^{10}$ Since the surface energy must be the same order as the total energy, $C$ must not be much smaller than unity. Investigation into what range of $C$ is allowed to stabilize the Fermi-ball is underway 43 .
} 
stringent constraints. We also noted the importance of future $T A$ and $O A$ experiments in the search for Fermi-balls as seen in Figure](b).

We lastly note that the charged solitons with a relatively light mass, $M \lesssim 10^{8} \mathrm{GeV}$, on which we have not focussed our attention, have astrophysical difficulties. If we consider that the dark matter consists mainly of such solitons with large cross section with matter and radiation, we will be faced by difficulties including 'the Galaxy-halo in-fall', 'too much heating of disk molecules', and 'too small density fluctuations in the early universe' 44,45. Therefore, We must show an interest in the window for the heavier charged solitons.

From these experimental bounds, we comprehensively obtained the stringent limitations on the properties of Q-balls and the Fermi-balls and then noted the possible importance of future experiments of $T A$ and $O A$. These bounds will help us study the unsolved cosmological problems that we have mentioned in this paper, by developing more realistic scenarios. In these cases, Q-balls and/or Fermi-balls play an essential role.

\section{Acknowledgement:}

We thank H.Tawara, M.Fukugita, M.Fukushima, and M.Sasaki for useful comments.

[1] S. Coleman, Nucl. Phys. B262 (1985) 263.

[2] A. Kusenko and M. Shaposhnikov, Phys. Lett. B418 (1998) 46.

[3] A. L. Macpherson and M. A. Campbell, Phys. Lett. B347 (1995) 205.

[4] J. R. Morris, Phys. Rev. D59 (1999) 023513.

[5] B. Holdom, Phys. Rev. D36 (1987) 1000 ; B. Holdom and R. A. Malaney, Astrophys. J. 420 (1994) L53.

[6] K. Enqvist and J. MacDonald, Phys. Lett. B425 (1998) 309.

[7] R. Brandenberger, I. Halperin, and A. Zhitnitsky, hep-ph/9903318.

[8] S. Kasuya and M. Kawasaki, hep-ph/9909509; hep-ph/0002285.

[9] A. Kusenko, M. Shaposhnikov, P. G. Tinyakov, and I. I. Tkachev, Phys. Lett. B423 (1998) 104.

[10] A. Kusenko, V. Kuzmin, M. Shaposhnikov, and P. G. Tinyakov, Phys. Rev. Lett. 80 (1998) 3185.

[11] I. A. Belolaptikov et al., astro-ph/9802223.

[12] E. N. Alexeyev et al., LETTERE AL NUOVO CIMENTO, 35 (1982) 413.

[13] P. B. Price and M. H. Salamon, Phys. Rev. Lett. 56 (1986) 1226.

[14] D. Bakari et al., hep-ex/0003003.

[15] Donald E. Groom, Phys. Rep. 140 (1986) 323 ; V. V. Ianovski et al., hep-ph/9909528 ; Particle Data Group, Review of Particle Physics, Eur. Phys. J. C3 (1998) 741, and references therein.

[16] E. K. Shirk and P. B. Price, The Astrophys. J. 220 (1978) 719.

[17] A. Kusenko, Phys. Lett. B406 (1997) 26.

[18] E. Witten, Phys. Rev. D30 (1984) 272.

[19] A. De Rújula and S. L. Glashow, NATURE 312 (1984) 734.

[20] V. A. Rubakov, Pis'ma Zh. Eksp. Teor. Fiz. 33 (1981) 658 ; JETP Lett. 33 (1981) 644 ; C. G. Callan, Jr. Phys. Rev. D26 (1982) 2058.

[21] J. Arafune and M. Fukugita, Phys. Rev. Lett. 50 (1983) 1901.

[22] T. Kajita et al., Journal of the Physical Society of Japan 54 (1985) 4065.

[23] Fabrizio Cei for the MACRO Collaboration, hep-ex/9810012.

[24] M. Ambrosio et al., Eur. Phys. J. C13 (2000) 453.

[25] S. Barwick et al., Contribution to Proc. of Astrophysical Aspects of the Most Energetic Cosmic Rays, Japan, Nov. 1990.

[26] AMANDA Collaboration, Proceedings of ICRC 99 (held at Salt Lake City, 17-25 Aug. 1999), ed. by D.Kieda et al., Vol 2 (1999) 344, and references therein.

[27] M. Fukushima, a talk at the 4th International RESCEU Symposium, - The Birth and Evolution of the Universe - Nov. 6-19, 1999 at Tokyo, the Proceedings are to be published ; Y.Arai et al., Proceedings of the 26th International Cosmic Ray Conference, Salt Lake City, Aug. 17-25, Vol.5 (1999) 393.

[28] M. Sasaki, private communications.

[29] J. Lindhard and M. Scharff, Phys. Rev. 124 (1961) 128, and references therein.

[30] J. A. Phipps et al., Phys. Rev. A36 (1964) 135.

[31] Gilbert L. Cano, Phys. Rev. 169 (1968) 277.

[32] W. D. Wilson, L. G. Haggmark, and J. P. Biersack, Phys. Rev. B15 (1977) 2458. 
[33] D. J. Ficenec et al., Phys. Rev. D36 (1987) 311.

[34] Needless to say, the precise estimation of flux limits or mass bounds of such experiments should be given by the experiment group by themselves with careful examination of the efficiencies of their detectors. We give here only the order of magnitudes though we think even such rough estimations may help some of those who are interested in Q-ball's flux. In case of the experiments of $O Y A$ [35] and NORIKURA [40], one of the present authors, S.Nakamura, is a member of the experiment group, who is rather familiar with these experiments.

[35] S. Orito et al., Phys. Rev. Lett. 66 (1991) 1951.

[36] T. Doke et al., Phys. Lett. B129 (1983) 370.

[37] K. Nakamura et al., Phys. Lett. B161 (1985) 417.

[38] T. Hara et al., Phys. Rev. Lett. 56 (1986) 553.

[39] K. N. Buckland et al., Phys. Rev. D41 (1990) 2726.

[40] S. Nakamura et al., Phys. Lett. B263 (1991) 529.

[41] J. Alcaraz et al., Phys. Lett. B461 (1999) 387 ; J. Derkaoui et al., Astropart. Phys. 9 (1998) 173.

[42] L. Scari, Proc. of 26th International Cosmic Ray Conference (Aug. 17-25, 1999 at Salt Lake City) ed. D. Kieda et al., Vol.2 (1999) 384.

[43] J. Arafune, K. Ogure, and T. Yoshida, in preparation.

[44] G.D.Starkman, A. Gould, R. Esmailzadeh, and S. Dimopoulos, Phys. Rev. D41 (1990) 3594.

[45] A. De Rújula, S. L. Glashow, and Uri Sarid, Nucl. Phys. B333 (1990) 173. 


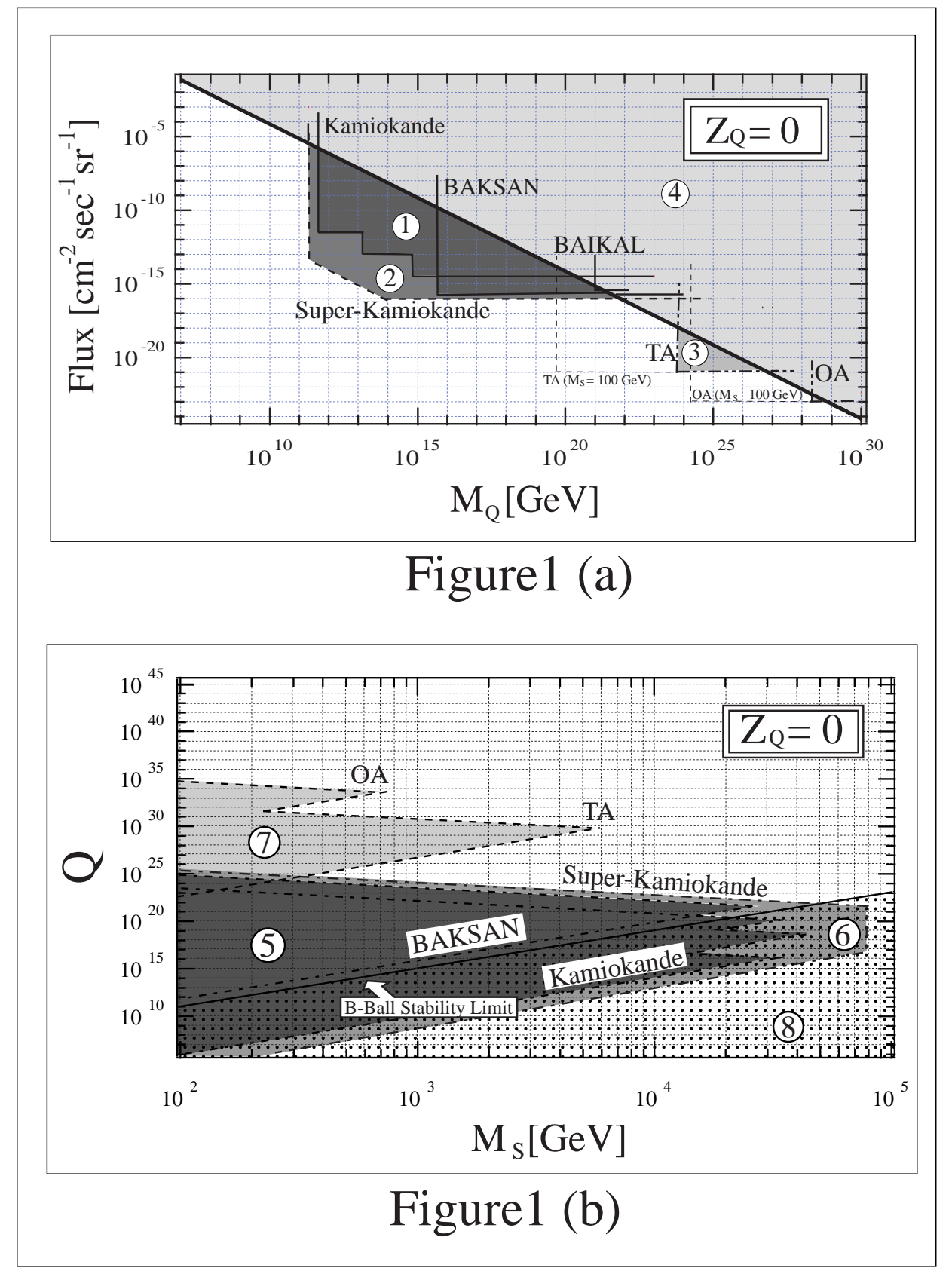

FIG. 1. Figure日(a) shows the bounds on flux and mass for neutral Q-balls $\left(Z_{Q}=0\right)$, i.e. SENS. The diagonal line shows the flux expected in case that the Galaxy dark matter $\left(\sim 0.3 \mathrm{GeV} / \mathrm{cm}^{3}\right)$ consists mainly of SENS, and thus the region above this DM-line (region 4), should be excluded. The region 1 is excluded by present and past experiments such as Gyrlyanda [1], $B A K S A N$ [12], and Kamiokande [22, which give us an accessible analysis for monopole search, as well as the region $\mathbf{2}$ by the Super-Kamiokande experiments, the data of which has not been analysed for the purpose of monopole search. The regions to be searched by future experiments such as $T A$ [27,28] and $O A$ [42], are also shown (region 3). The lower mass bound in each experiment is calculated taking $M_{S}=1 \mathrm{TeV}$ (taking also $M_{S}=100 \mathrm{GeV}$ for $T A$ and $O A$ ). Figure1 (b) shows bounds on the $\mathrm{U}(1)$ charge $Q$ versus the symmetry breaking scale $M_{S}$ for SENS $\left(Z_{Q}=0\right)$ in the case of the Galaxy dark matter consisting mainly of SENS (the flux and mass of SENS in this case is restricted to lie on the diagonal line of Figure1 (a)). The region 5, $\mathbf{6}$, and $\mathbf{7}$ are searched in the same experiments as the region 1, 2, and 3, respectively. The line with "B-Ball Stability Limit" shows that the region below it (region 8) is not allowed to have the stability of SENS in case they are "B-balls" (as discussed in the text). The allowed region $Q>10^{22}$ is only the upper blank part of the figure. 


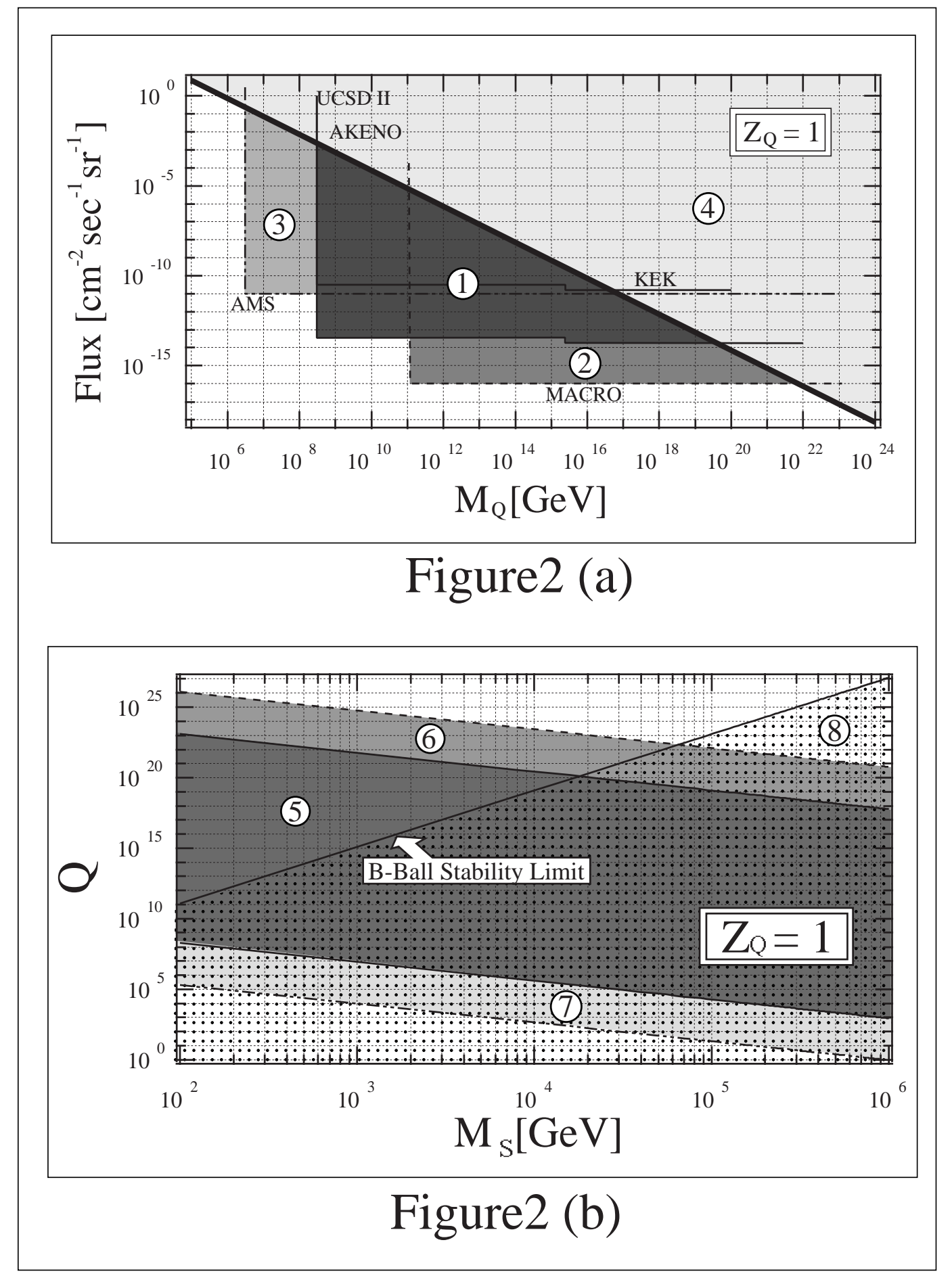

FIG. 2. Figure2(a) shows limits on the Q-ball's flux and its mass for SECS with $Z_{Q}=1$. The diagonal line is the same as in Figure1 (a) and the region above this line (region 4), should be excluded. The region 1 is excluded by present and past experiments such as KEK 37], AKENO 38, and UCSDII [39], which give us an accessible analysis for monopole search, as well as the region 2 by the MACRO 14,23. 24 experiments, the data of which has not been analysed for monopole search yet. The regions to be searched by future experiments such as $A M S$ [41], are also shown (region 3). Figure. (b) represents bounds on $Q$ and $M_{S}$ of SECS with $Z_{Q}=1$ (see texts for details). The marks and patterns to separate regions are the same as those used in Figure(b). 


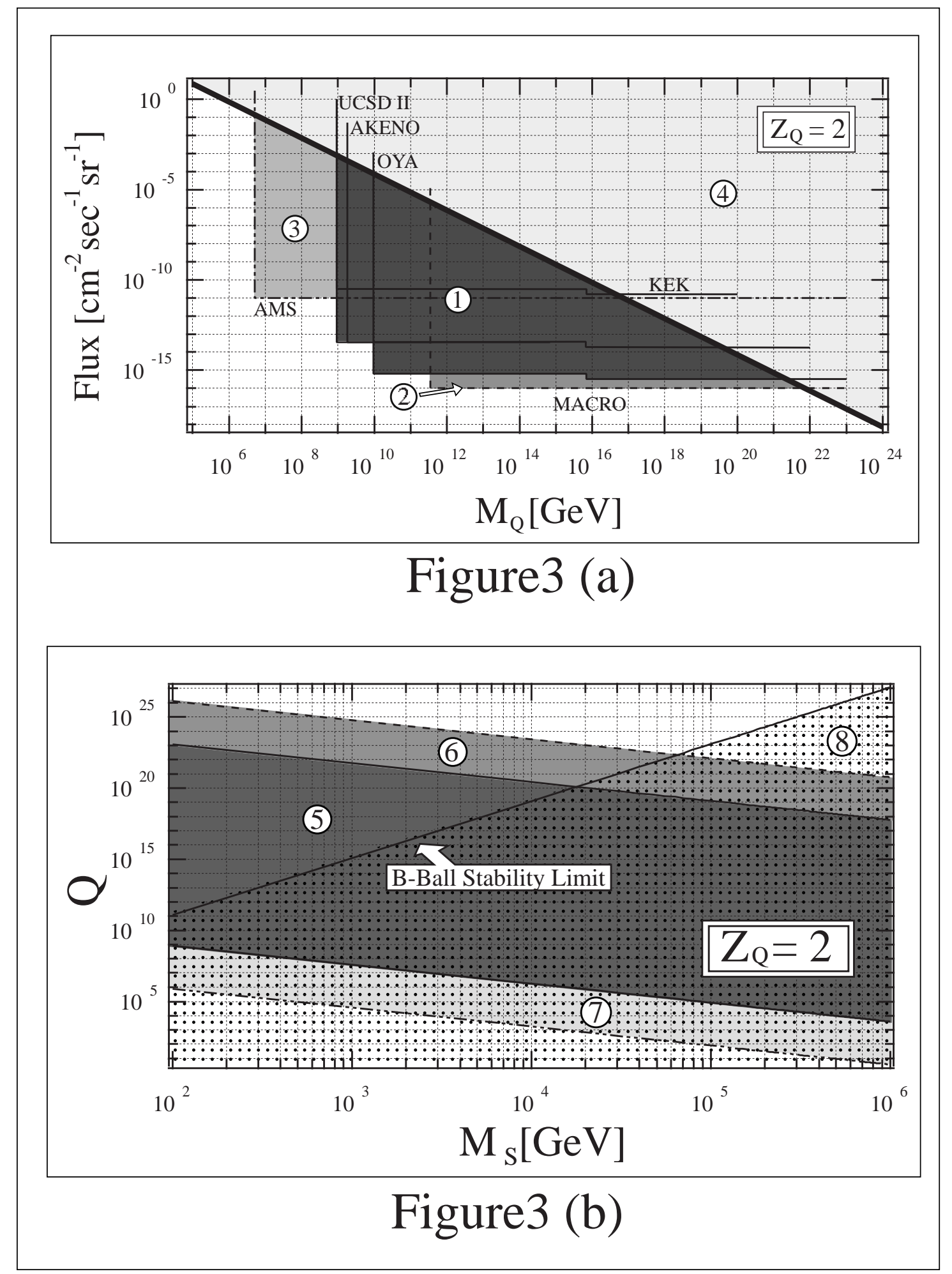

FIG. 3. Figure 3 (a) shows limits on SECS with $Z_{Q}=2$. This figure is similar to Figure 2 (a) except that the values of the mass has lower limits and the $O Y A$ 35] experiments are included. Figure 3 (b) represents limits on $Q$ and $M_{S}$ of SECS with $Z_{Q}=2$. The marks and patterns to separate regions are the same as those used in Figure2(b). 


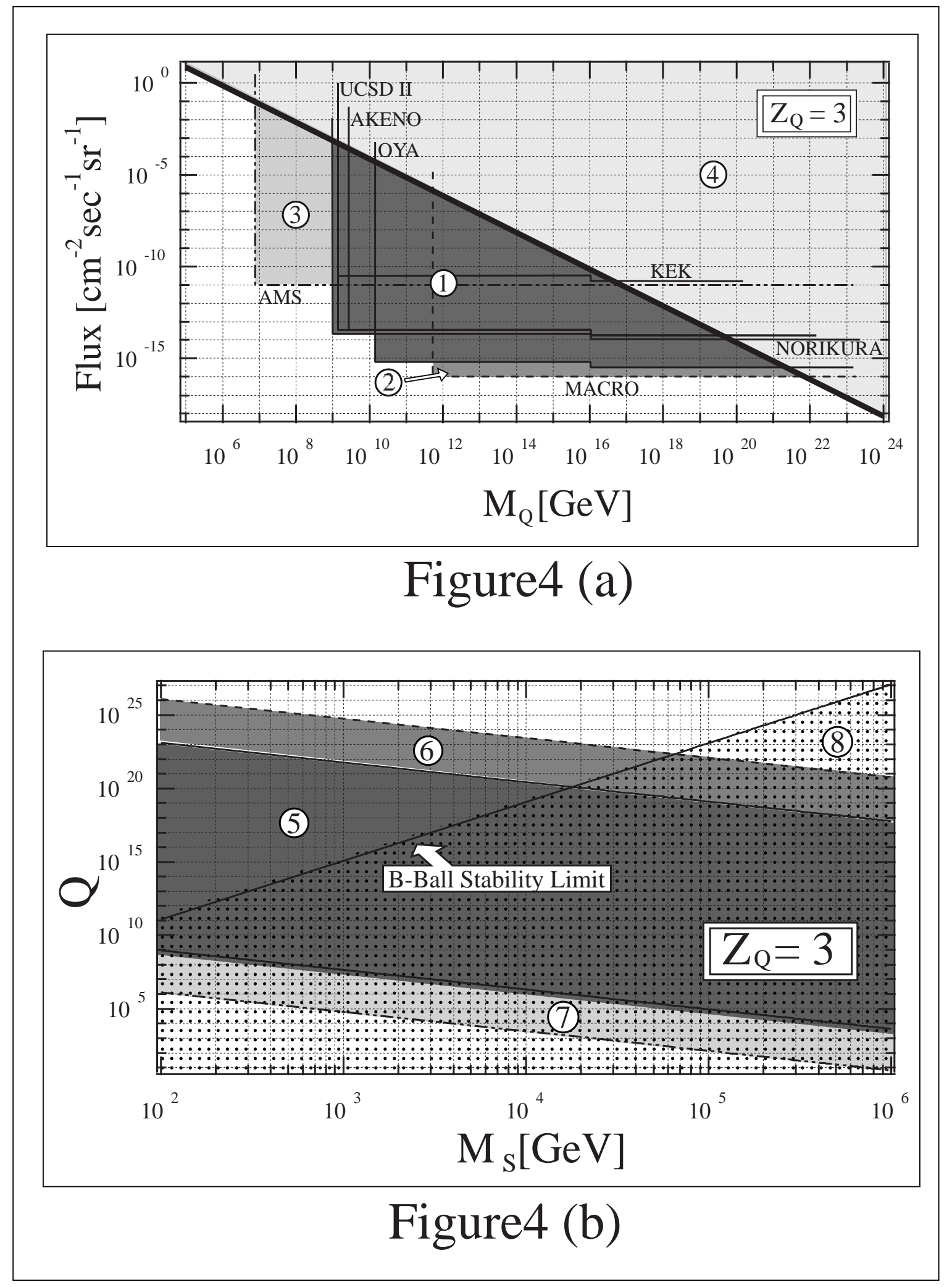

FIG. 4. Figure 4 (a) shows limits on SECS with $Z_{Q}=3$. This figure is similar to Figure 3 (a) except that the values of the mass has lower limits and the NORIKURA 40] experiments are included. Figure屯/(b) represents bounds on $Q$ and $M_{S}$ of SECS with $Z_{Q}=3$. The marks and patterns to separate regions are the same as for those used in Figure 3 (b). 


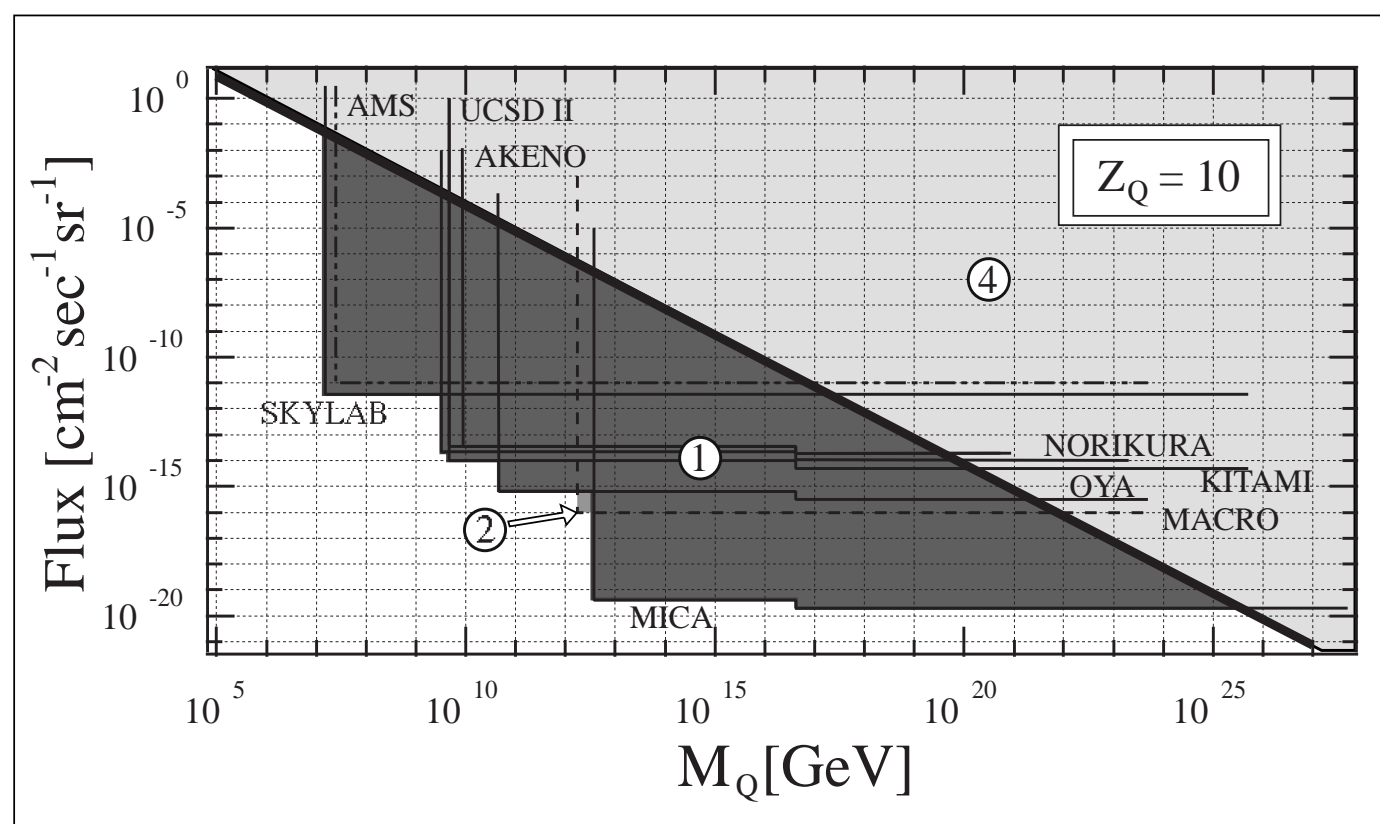

\section{Figure5 (a)}

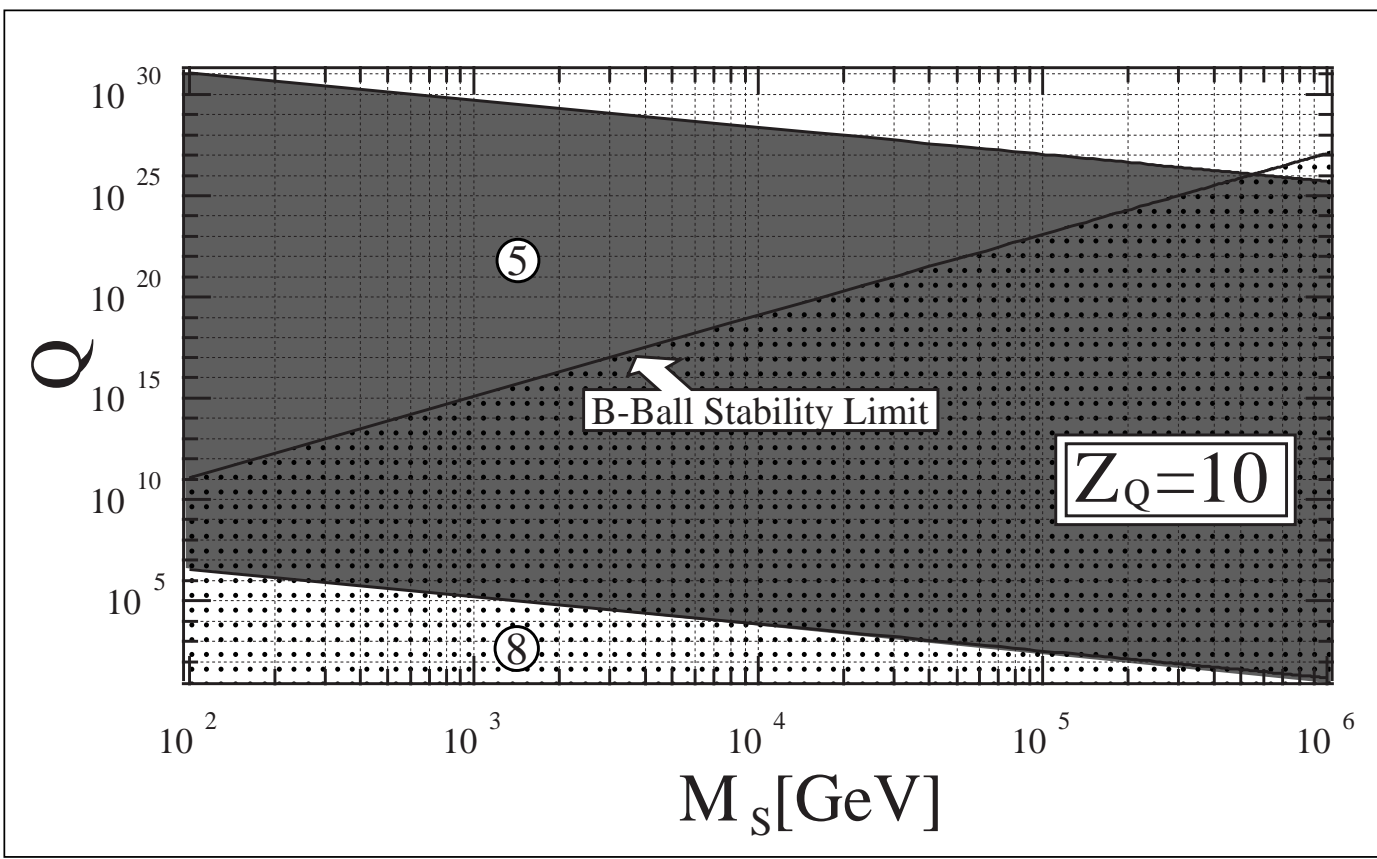

Figure5 (b)

FIG. 5. Figure- (a) shows limits on SECS with $Z_{Q}=10$. This figure is similar to Figure国(a) except that the values of mass has lower limits and the experiments of SKYLAB [16], KITAMI 36, and MICA [13] are included. The experiments of MICA and $S K Y L A B$ give stringent limits to the SECS. Figure5(b) represents limits on $Q$ and $M_{S}$ of SECS with $Z_{Q}=10$. The marks and patterns to separate regions are the same as for those used in Figure白(b). 


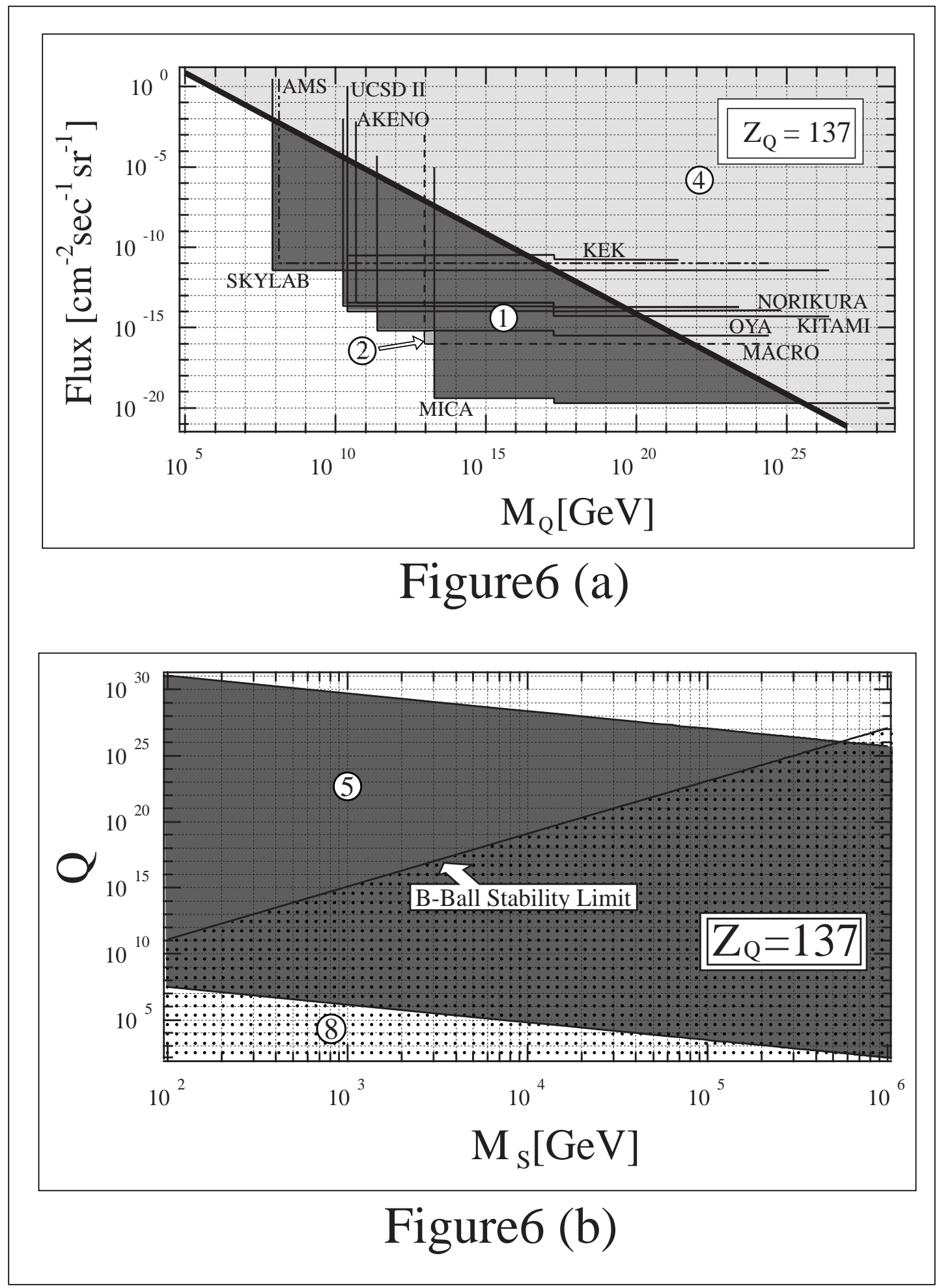

FIG. 6. Figure6(a) shows bounds on SECS with $Z_{Q}=137$. This figure is similar to Figuref(a) except for the values of the excluded mass region. In this case the cross section for the collision with matter atoms are assumed to be $\pi R_{e f f}^{2}$ with $R_{\text {eff }} \sim 1 \AA$. We expect that the case where $Z_{Q}>137$ is the same as the case where $Z_{Q}=137$. Figure 6 (b) represents limits on $Q$ and $M_{S}$ of SECS with $Z_{Q}=137$. The marks and patterns to separate regions are the same as for those used in Figureßf(b). 


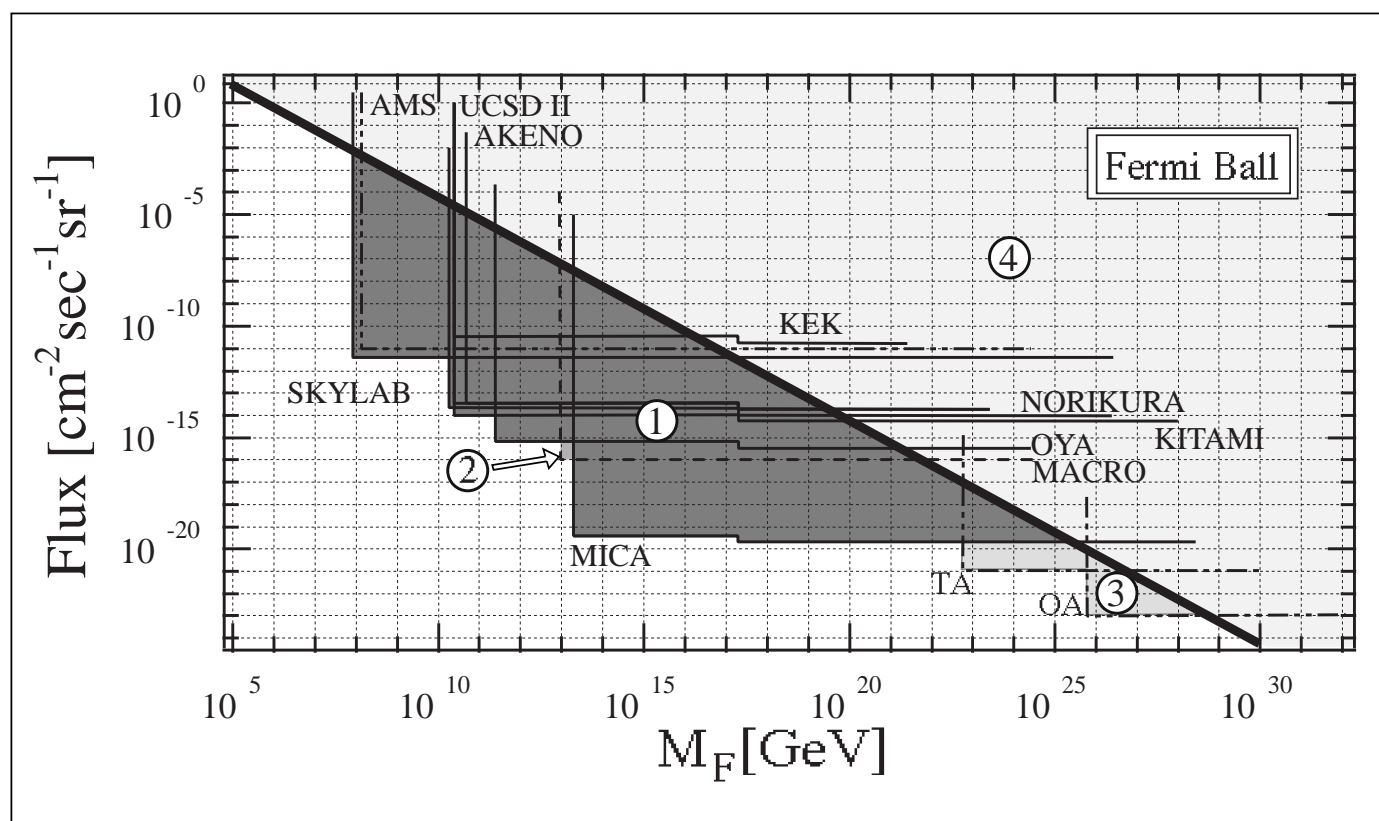

\section{Figure7 (a)}

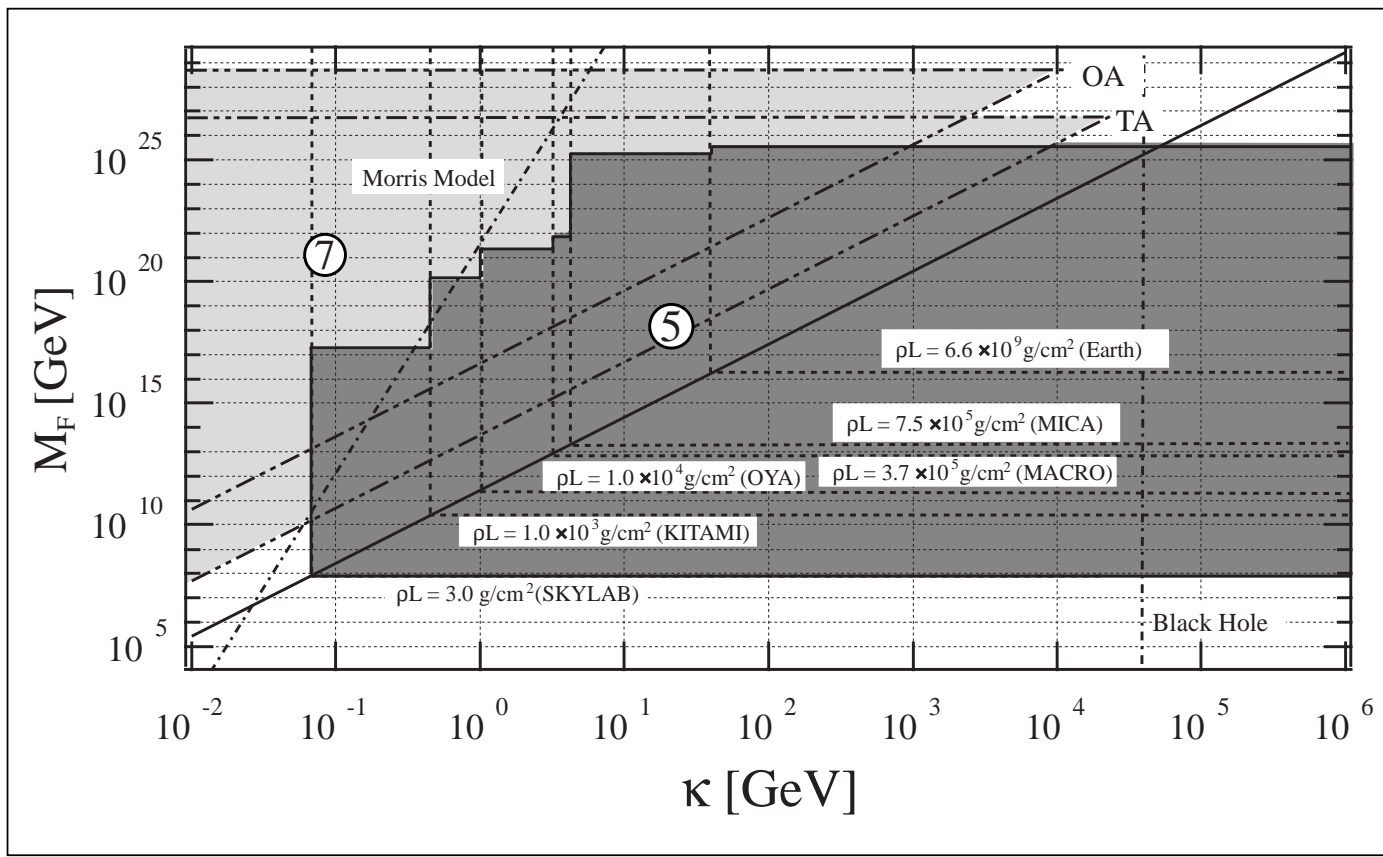

Figure7 (b)

FIG. 7. Figure (a) shows the limits of flux and mass of Fermi-balls obtained from various experiments. This is similar to Figure (a) (SECS with $Z_{Q}=137$ ), although it has additional restrictions to be expected in the future $T A$ and $O A$ experiments. Figuref(b) represents an excluded region in the $M_{F}-\kappa$ plane. The region $\mathbf{5}$ is excluded by the available data of present and past experiments. The region 7 is to be investigated by future experiments such as TA and $O A$. The Morris's Model line(Eq.(37)) and black hole limit(Eq.(38)) are also shown in this figure (taking $C=1$ ). 